Investigation of positive streamers by double-pulse experiments, effects of repetition rate and gas mixture

This content has been downloaded from IOPscience. Please scroll down to see the full text.

2014 Plasma Sources Sci. Technol. 23025008

(http://iopscience.iop.org/0963-0252/23/2/025008)

View the table of contents for this issue, or go to the journal homepage for more

Download details:

IP Address: 131.155.2.68

This content was downloaded on 27/03/2014 at 13:18

Please note that terms and conditions apply. 


\title{
Investigation of positive streamers by double-pulse experiments, effects of repetition rate and gas mixture
}

\author{
S Nijdam ${ }^{1}$, E Takahashi ${ }^{2}$, A H Markosyan ${ }^{3}$ and U Ebert ${ }^{1,3}$ \\ ${ }^{1}$ Department of Applied Physics, Eindhoven University of Technology, PO Box 513, 5600 MB \\ Eindhoven, The Netherlands \\ ${ }^{2}$ National Institute of Advanced Industrial Science and Technology (AIST), 1-2-1 Namiki, Tsukuba \\ Ibaraki 305-8564, Japan \\ ${ }^{3}$ Centrum Wiskunde \& Informatica (CWI), Amsterdam, The Netherlands \\ E-mail: s.nijdam@tue.nl
}

Received 16 October 2013, revised 20 November 2013

Accepted for publication 30 December 2013

Published 19 March 2014

\begin{abstract}
Streamer discharges are often operated in a repetitively pulsed mode and are therefore influenced by species left over from the previous discharge, especially free electrons and ions. We have investigated these effects by applying two consecutive positive high voltage pulses of 200-700 ns duration to a point-plane gap in artificial air, pure nitrogen, other nitrogen-oxygen mixtures and pure argon at pressures between 67 and 533 mbar. The pulses had pulse-to-pulse intervals $(\Delta t)$ between $200 \mathrm{~ns}$ and $40 \mathrm{~ms}$. We imaged both discharges with two ICCD cameras and combined this to a compound image. We observe for values of $\Delta t$ below $0.5-15 \mu \mathrm{s}$ (at 133 mbar, with $\Delta t$ depending on gas mixture) that during the second pulse the streamers continue the paths of the first-pulse streamers. We call the maximal time for which this continuation still occurs the continuation time. For $\mathrm{N}_{2}-\mathrm{O}_{2}$ mixtures, this time has a maximum at an oxygen concentration of about $0.2 \%$. According to our plasma-chemical modelling this maximum is determined by the electron loss rate which has a minimum around this oxygen concentration. Depending on oxygen concentration the dominant recombining positive ion is $\mathrm{N}_{4}^{+}, \mathrm{O}_{2}^{+}$or $\mathrm{O}_{4}^{+}$where $\mathrm{O}_{2}^{+}$dominates around $0.2 \% \mathrm{O}_{2}$ and recombines slowest.

For increasing values of $\Delta t$ we observe that after the continuation phase first no new streamers occur at all, then new streamers show up that avoid the entire pre-ionized region. Next we see new thin streamers that follow the edges of the old channels. For larger $\Delta t$ $(10-200 \mu \mathrm{s})$ the new streamers start to increase in size and move to the centre of the old channels. Finally, around millisecond timescales the new channels are completely independent of the old channels.

Together this points to the combination of two mechanisms: streamers search the proximity of regions with increased electron density, but cannot penetrate regions with very high electron density.
\end{abstract}

Keywords: streamer, pulse, background ionization density, nitrogen, oxygen

(Some figures may appear in colour only in the online journal)

S Online supplementary data available from stacks.iop.org/PSST/23/025008/mmedia

\section{Introduction}

It has been observed by many groups that streamer discharges are in many ways influenced by preceding discharges [1-4].
From these and other studies, it is clear that species produced by such discharges play an important role in the inception and propagation of streamers. In most cases it is assumed that the dominant effect of preceding discharges is the creation 
of (background) ionization. Immediately after a discharge the background ionization consists primarily of free electrons and positive ions, depending on composition and pressure. For longer timescales this generally changes to positive and negative ions due to electron attachment and/or recombination, again depending on composition and pressure. When the positive and negative species are not distributed equally this will lead to space-charge which can also influence subsequent streamer discharges by its resulting electric field. Other species that can play a role in addition charged species are radicals (e.g. $\mathrm{N}$ and $\mathrm{O}$ ), metastables (e.g. $\mathrm{N}_{2}\left(A^{3} \Sigma_{\mathrm{u}}^{+}\right)$and $\left.\operatorname{Ar}(4 \mathrm{~s})\right)$ as well as other products like $\mathrm{NO}$ and $\mathrm{OH}$.

One way of studying the effects of initial conditions on a streamer discharge is to create background ionization with an independent source like a laser [5], x-rays [6] or radioactive compounds [1]. This has the advantage that it decouples the production of ionization (and other important long-lived species) from the streamers.

Here, we will investigate the effects of a preceding streamer discharge on a subsequent discharge by applying two positive high voltage pulses in succession with pulse-topulse intervals $(\Delta t)$ between $200 \mathrm{~ns}$ and $40 \mathrm{~ms}$. This allows us to study the effect of the preceding discharge on a very large range of time scales. At low values of $\Delta t$ the time scale is of similar order as the characteristic time of the total streamer development and the duration of the applied pulse. Therefore, we can also learn things about streamer development in general. Values of $\Delta t$ around $100 \mu \mathrm{s}$ to $1 \mathrm{~ms}$ correspond to repetitive streamer discharges with $1-10 \mathrm{kHz}$ repetition frequency that are often used in commercial streamer and plasma-jet applications [7-9]. Finally, at $\Delta t \geqslant 10 \mathrm{~ms}$ the discharges start to resemble the 'single pulse' discharges often used to study streamer development.

The advantage of applying two pulses instead of a continuous pulse train is that it is much easier to see the effects of a single pulse discharge on the next discharge and that it allows the huge range of $\Delta t$ that was discussed above. It is experimentally extremely challenging to build a pulse source with a variable repetition frequency between $10 \mathrm{~Hz}$ and $50 \mathrm{MHz}$ while maintaining the same pulse shape and amplitude. Creation of two identical pulses with variable interval is much easier.

Walsh et al $[10,11]$ have shown in experiments with atmospheric pressure plasma-jets in helium that, for jets extending beyond the guiding tube, the propagation can be temporarily halted by 'neutralizing' the applied voltage by applying the same voltage to two external electrodes surrounding the jet. This resembles the application of two consecutive pulses to a single electrode as in our experiments.

In this paper, we will discuss the following topics: in section 2 we give the details and parameters of our experiment and methods. Section 3 treats the general development of streamer discharges created with two pulses as a function of the time gap $\Delta t$ between pulses by means of ICCD imaging. This is done for three gases: artificial air, pure nitrogen and pure argon for various pressures and pulse widths and amplitudes. In section 4 , we focus on the value of $\Delta t$ for which we can still observe continuation of the first-pulse streamers during

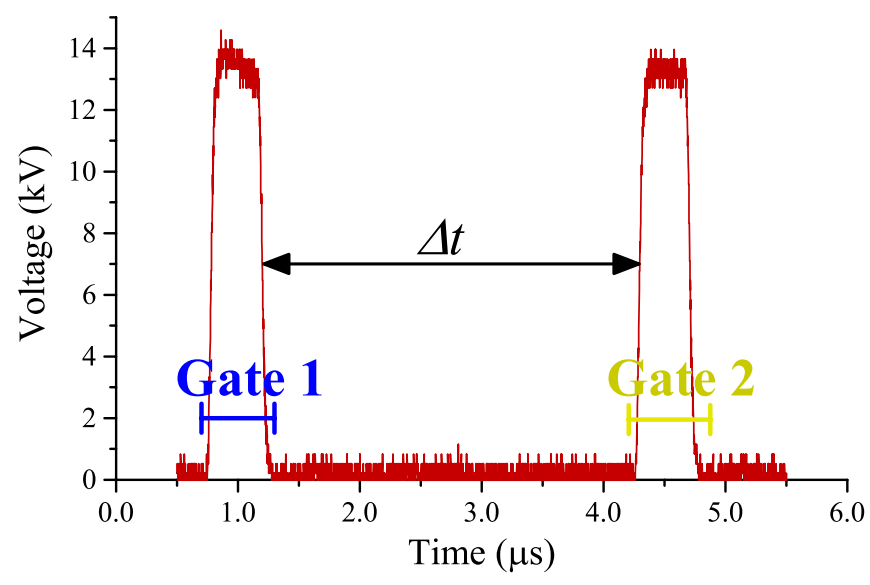

Figure 1. Typical shape of the two subsequent voltage pulses with the pulse-to-pulse interval $\Delta t$ indicated. Indications of the opening (gate) times of the two different ICCD cameras are shown in blue and yellow, respectively.

the second pulse and how this depends on gas conditions, most notably on the variation of the oxygen concentration in nitrogen-oxygen mixtures. The experimental results are then compared with plasma-chemical modelling results in section 5 . Finally, we will summarize our results and draw conclusions in section 6 .

\section{Experimental set-up}

All experiments are carried out in a $103 \mathrm{~mm}$ point-plane gap mounted inside a vacuum vessel. This vessel is filled with artificial air, pure nitrogen, pure argon and air-nitrogen mixtures at pressures between 67 and 533 mbar. When using pure nitrogen or argon, the vessel is flushed at a rate of roughly 1 standard litre per minute to improve gas purity in the experiments. The purity of all of the source gases is specified as 6.0 (less than 1 ppm impurities). However, because of leakage and/or out-gassing, we estimate the impurity level during experiments at about $5 \mathrm{ppm}^{4}$. On the pointed tungsten tip with a tip radius of $15 \mu \mathrm{m}$, a pair of positive voltage pulses is applied repeatedly. Such a pulse pair consists of two nearly identical pulses with pulse-to-pulse intervals $(\Delta t)$ between $200 \mathrm{~ns}$ and $40 \mathrm{~ms}$. The pulse pairs are applied with a repetition frequency of $0.7 \mathrm{~Hz}$. The individual pulses that make up the pulse pairs are relatively rectangular shaped with pulse lengths of 200-700 ns, rise/fall times of about $15 \mathrm{~ns}$ and pulse amplitudes of 7-17kV. An example of such a pulse pair is shown in figure 1, where the definition of $\Delta t$ is indicated as well. Pulse amplitudes are here defined as the average voltage of the plateau during the first pulse. For small values of $\Delta t$ the amplitude during the second pulse can be slightly smaller, as shown in figure 1.

The streamer discharges induced by the two voltage pulses are imaged by two separate, nearly identical ICCD cameras. These cameras are mounted so that they image exactly the same plane, by means of a half-mirror (see figure 2). During

4 This was calculated by dividing the measured leak/out-gassing rate of $5 \times 10^{-6}$ standard litres per minute by the applied gas flow of 1 standard litre per minutes. 


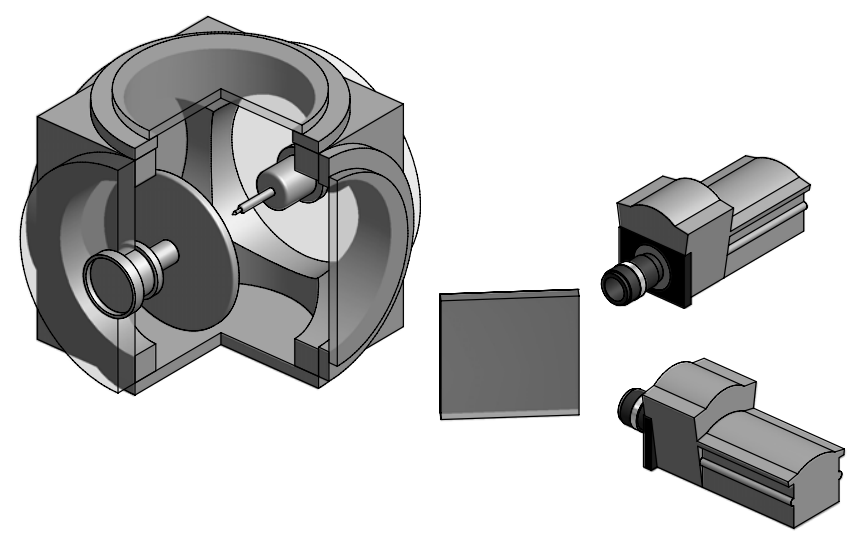

Figure 2. Schematic illustration of vacuum vessel, half-mirror and two ICCD cameras. Part of the vacuum vessel has been cut from the rendering to show the tip and plate. In all presented images the recorded images are rotated anti-clockwise by $90^{\circ}$ so that the electrode tip is shown on top.

post-processing the images are rotated so that the electrode tip is always located at the top of the image. The gate of one camera is opened during the first voltage pulse; the gate of the other camera is opened during the second voltage pulse (see gate indications in figure 1).

A typical example of an image-pair produced by the two cameras is shown in figures $3(a)$ and $(b)$, where the images are acquired during the first and second pulse, respectively. These two images are then laid over each other as a new image where the blue channel (component) of the RGB-pixels represents the first pulse image and the red and green channels represent the second pulse image. A pixel with the red and green channels equally bright and the blue channel off will appear yellow, while a pixel with all three channels fully illuminated will appear white. Therefore, areas that only emit during the first pulse are blue, areas that only emit during the second pulse are yellow and areas that emit during both pulses are white. All linear combinations of yellow and blue are possible. The result of this operation in figures 3(a) and $(b)$ is shown in figure 3(c).

In the example from figures $3(a)-(c)$ it is clear that the (blue) streamer channels created by the first pulse continue their path (in yellow) during the second pulse. The area around the electrode tip, as well as some areas on the edge of the inception cloud emit again during the second pulse and are therefore coloured white.

Figure $3(d)$ shows a superimposed image with both cameras gated around the first pulse (under different conditions than in figures $3(a)-(c))$. In this case both cameras should capture exactly the same image. This shows that the two cameras are aligned properly as almost all streamer channels are rendered as white or grey and only a slight yellow/blue offset is visible.

Note that in all images presented in this paper the intensities of both cameras are chosen arbitrarily. The same intensity on the image does not imply that the original streamers have the same intensity. This was done for several reasons: firstly, the two cameras are slightly different and do not have the same gain factors. Secondly, there was a half-mirror between the vessel and the cameras which has different losses
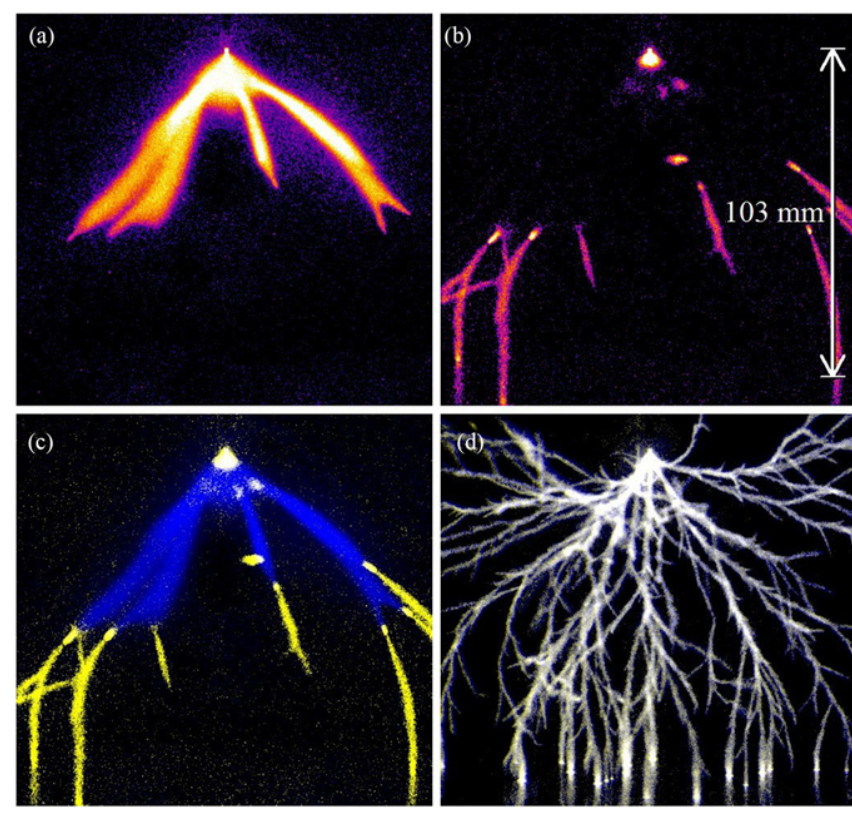

Figure 3. Illustration of the two-camera overlay technique; $(a)$ and (b) show false-colour representations of the captured frames from camera 1 and 2, respectively, recorded during one voltage pulse pair (conditions: $6.8 \% \mathrm{O}_{2}$ in $\mathrm{N}_{2}, 133$ mbar gas, $13.6 \mathrm{kV}, 200$ ns pulses with $\Delta t=500 \mathrm{~ns})$. In (c) the images from $(a)$ and $(b)$ are superimposed in blue and yellow, respectively. $(d)$ shows a similar overlay of a discharge under different conditions, with both camera gate times set at the first pulse only (conditions: pure $\mathrm{N}_{2}, 133 \mathrm{mbar}$ gas, $13.6 \mathrm{kV}, 400 \mathrm{~ns}$ pulse).

for transmission and reflection and finally because the streamer morphology is best imaged with the maximum contrast per image. For these reasons we have used automatic contrast and brightness algorithms to process the final output images. This may also explain the slight blue tint in figure $3(d)$.

In all cases, observations are performed on twodimensional images of the three-dimensional discharge structures. Therefore, it is not always possible to draw conclusions on the exact relation between first and second discharge from one image. To do this properly one would need a stereoscopic set-up [12] to observe the full three-dimensional relation between the paired images. Nevertheless, observation of multiple images under the same conditions can give good insight in the morphology and the relation between the paired discharges. All observations discussed in this paper are based upon multiple images per parameter setting (usually 5 or 10 images), but only a limited subset of these images is presented here.

\section{General development}

We have studied the morphology of the discharge induced by the voltage pulse pairs as a function of pulse-to-pulse interval $(\Delta t)$ for a variety of gases, pressures and voltage pulse lengths and amplitudes. In general, the development during the second pulse as a function of $\Delta t$ is similar for all conditions, although there are variations in detail. We will first discuss the full development for one set of conditions in air. Next we will look into 

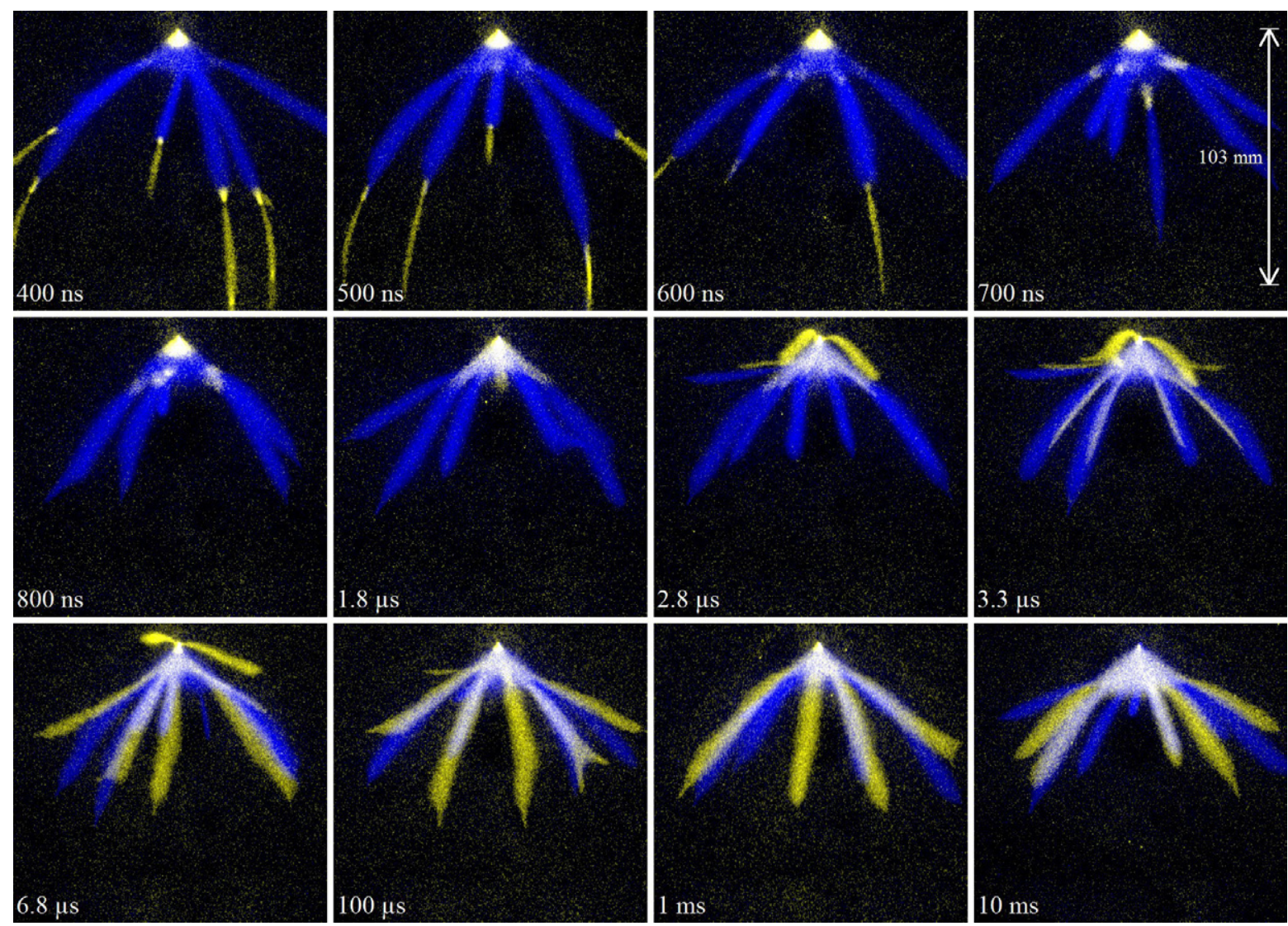

Figure 4. Superimposed discharge-pair images for varying $\Delta t$ as indicated in the images. Images taken in 133 mbar artificial air with pulses of $13.6 \mathrm{kV}$ amplitude and $200 \mathrm{~ns}$ pulse length.

the effects of pulse length, voltage and pressure on this development. Finally, we will look at pure nitrogen and pure argon.

Note that because the gate of the cameras is opened during the full duration of the two respective pulses, propagating streamer heads will be imaged as continuous trails [13-15]. The trails created during the first pulse will indicate the position of the channels of leftover species created during this pulse. For sub-millisecond timescales the effects of diffusion and convection on the location of the trails is expected to be minimal.

\subsection{Air}

An example of the development of the discharges during the two pulses in 133 mbar air with relatively short pulses and a large variation in $\Delta t$ is given in figure 4 . The discharges generated by the first pulse are, except for stochastic variations, similar for all values of $\Delta t$. These first-pulse discharges consist of a so-called inception cloud that breaks up into a few (typically five or six) streamer channels which mostly terminate either at the moment the first pulse is stopped or when the (bottom) cathode plate has been reached. Under the conditions from figure 4 the pulse duration has been chosen so that the streamers terminate roughly halfway between electrode tip and cathode plate.

We can describe the development of the discharge during the second pulse as a function of $\Delta t$ for the conditions of figure 4 with the following six stages: (i) At very short pulse-to-pulse intervals ( $\Delta t<700 \mathrm{~ns})$, the second-pulse streamers continue where the first-pulse streamers stopped at the end of the first pulse. At the new starting point a structure resembling a small scale inception cloud is visible. The extended streamer channels become shorter, thinner and fewer for increasing $\Delta t$. Furthermore, the regions around the tip and the positions where the first streamers originally emerged from the inception cloud both emit light again, as can be seen from their white colour.

(ii) At $\Delta t \geqslant 700 \mathrm{~ns}$ the first-pulse channels are no longer continued during the second pulse. Instead the re-glowing points at the edge of the inception cloud become more pronounced and eventually at $\Delta t=1.8 \mu$ s grow together with the emitting region around the tip.

(iii) At $\Delta t \geqslant 2.5 \mu \mathrm{s}$ the first new streamer channels start to appear. These channels avoid the entire old inception cloud region and move around it.

(iv) At $\Delta t=3.3 \mu \mathrm{s}$ more new channels appear. These new channels seem to follow the edges of the old channels. They are clearly thinner than both the old channels and the other new channels that avoid the inception cloud. Note that at $\Delta t=1.8 \mu \mathrm{s}$ a few short channels with similar morphology are also visible. Analysis of a large number of images under these conditions has shown a preference for the bottom side (with respect to the orientation of the presented images) of the old streamer channels. Images made with shorter gate times have revealed that 
these channels are the time-integrated representation of travelling dots (streamer heads) and not continuous reillumination of existing channels.

(v) At larger values of $\Delta t$ the new channels increase in width until they are roughly as wide as the original channels around $\Delta t=5.8 \mu \mathrm{s}$ and start to overlap more with the parent channels. For increasing $\Delta t$ they also start to become less dependent on the location of the first-pulse streamers. However, up to $\Delta t=1.0 \mathrm{~ms}$ a large number of the second-pulse streamers still follows the first-pulse streamer paths, although not always exactly. Under these conditions the inception cloud during the second pulse is significantly smaller than during the first pulse.

(vi) Around $\Delta t=10 \mathrm{~ms}$ the second discharge has become fully independent of the first discharge. The inception cloud sizes as well as the thickness and position distributions of the streamers are now the same for the two pulses. Furthermore, the position of the streamers during the second pulse no longer shows a correlation with the positions during the first pulse.

Note that these six stages cannot always be distinguished exactly and that the exact transition between stages is often difficult to pinpoint.

Interpretation. The structures that are visible in stage (i) resemble the findings of Walsh et al [10] where atmospheric pressure plasma-jets in helium also continue their path after the applied external field was suppressed for a short period. We will discuss the stage (i) streamer continuation and its interpretation in depth in sections 4 and 5.2.

The re-emission of light from some regions during stages (i)-(iv) is probably similar to a secondary streamer or glow discharge. This is in fact not a real propagating streamer front but instead re-illumination of a previously ionized area due to a change in field distribution. A more detailed investigation has revealed that during the second pulse the re-glowing of the points around the tip and the original inception cloud occurs early in the pulse, while the continuation of the old channels starts from about 50-100 ns after the pulse start. Note that it is very likely that parts of the old channels that do not re-emit during the second pulse still carry (dark) current. Because their conductivity is still high no high fields can exist and current will not produce any light. For larger $\Delta t$ the conductivity decreases and therefore the secondary streamers become more pronounced.

The new channels avoiding the old ones that appear in stage (iii) appear similar to the streamer morphology treated in [2], where new streamers avoid the pre-ionized area generated by a previous discharge. Similar behaviour was found by Briels et al [16] where long voltage pulses were applied to a point-plane gap in ambient air and so-called late streamers are visible that avoid the area occupied by earlier streamers. In both these cases as well as in the results presented here the new streamers move around the area covered by the preceding streamers or inception cloud. This is probably caused by the high conductivity in this area which will quickly shield any electric field and therefore inhibit the formation of an ionization front. The position of the new channels at
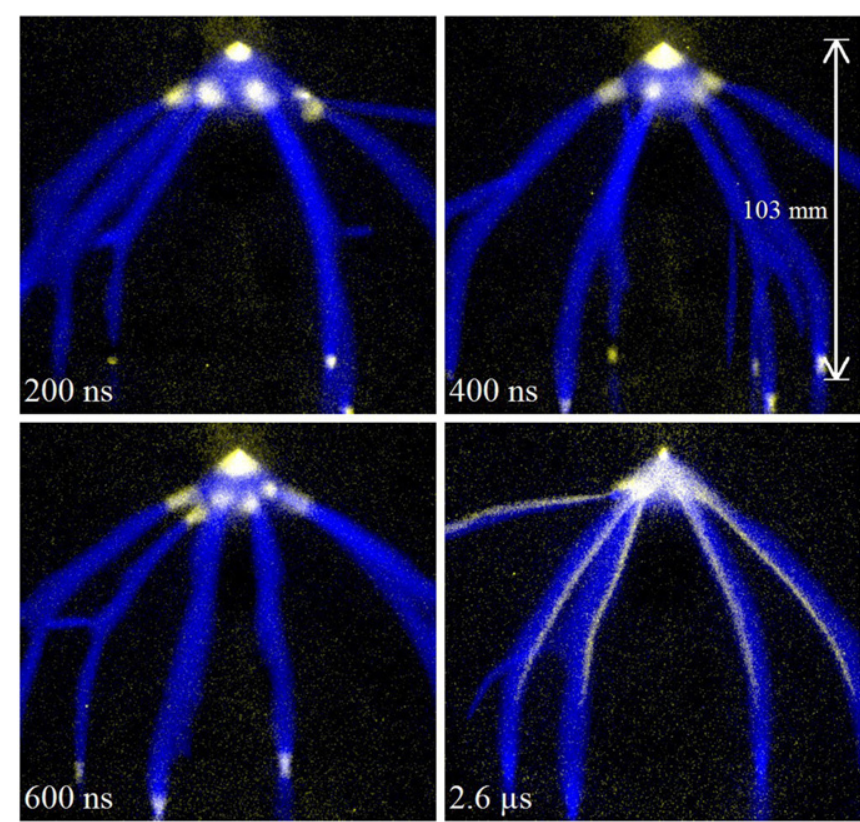

Figure 5. Superimposed discharge-pair images for varying values of $\Delta t$. Images taken in 133 mbar artificial air with pulses of $13.6 \mathrm{kV}$ amplitude and $400 \mathrm{~ns}$ length.

the edge of the old ones in stage (iv) seems to be related to this: the inside of the old channels is still very conductive for discharge propagation but the edges have an electron density which is preferable for streamer propagation. The preference for the bottom side of the channels may be due to the higher background field in this region (closer proximity to the cathode plane). Alternatively, as was proposed by Mark Kushner at the GEC 2013, the outer regions of the old channels may contain higher concentrations of $\mathrm{O}_{2}^{-}$and may therefore be more attractive for streamer propagation. In stage (v) the new channels can slowly penetrate the area of the old channels due to the decrease in conductivity. The guiding effect of the old channels on the new channels can also be partly due to an increase in temperature, and therefore a decrease in gas density, in the old channels. However, we do not expect a large temperature increase [17] so this effect will probably be minor.

Longer pulses. When $400 \mathrm{~ns}$ voltage pulses instead of $200 \mathrm{~ns}$ pulses are applied, the development of the second-pulse streamer as a function of $\Delta t$ is very similar (see figure 5). The only differences are related to the fact that the firstpulse streamers have already crossed the gap. Therefore, the occurrence of channel-continuation for small values of $\Delta t$ (stage (i)) is rare as this only occurs on the few branches that did not cross the channel during the first pulse. However, the branches that did cross show something else: during the second voltage pulse the cathode spots (the 'feet' of the channels) from the first discharge light up again. This effects lasts to about $\Delta t=1 \mu \mathrm{s}$. Furthermore, re-glowing of the spots at the edge of the original inception cloud is more pronounced with these longer pulses and the new channels start to occur for smaller values of $\Delta t$ than with the shorter pulse (compare $\Delta t=2.6 \mu \mathrm{s}$ from figure 5 to $\Delta t=2.8 \mu$ s from figure 4 ). 


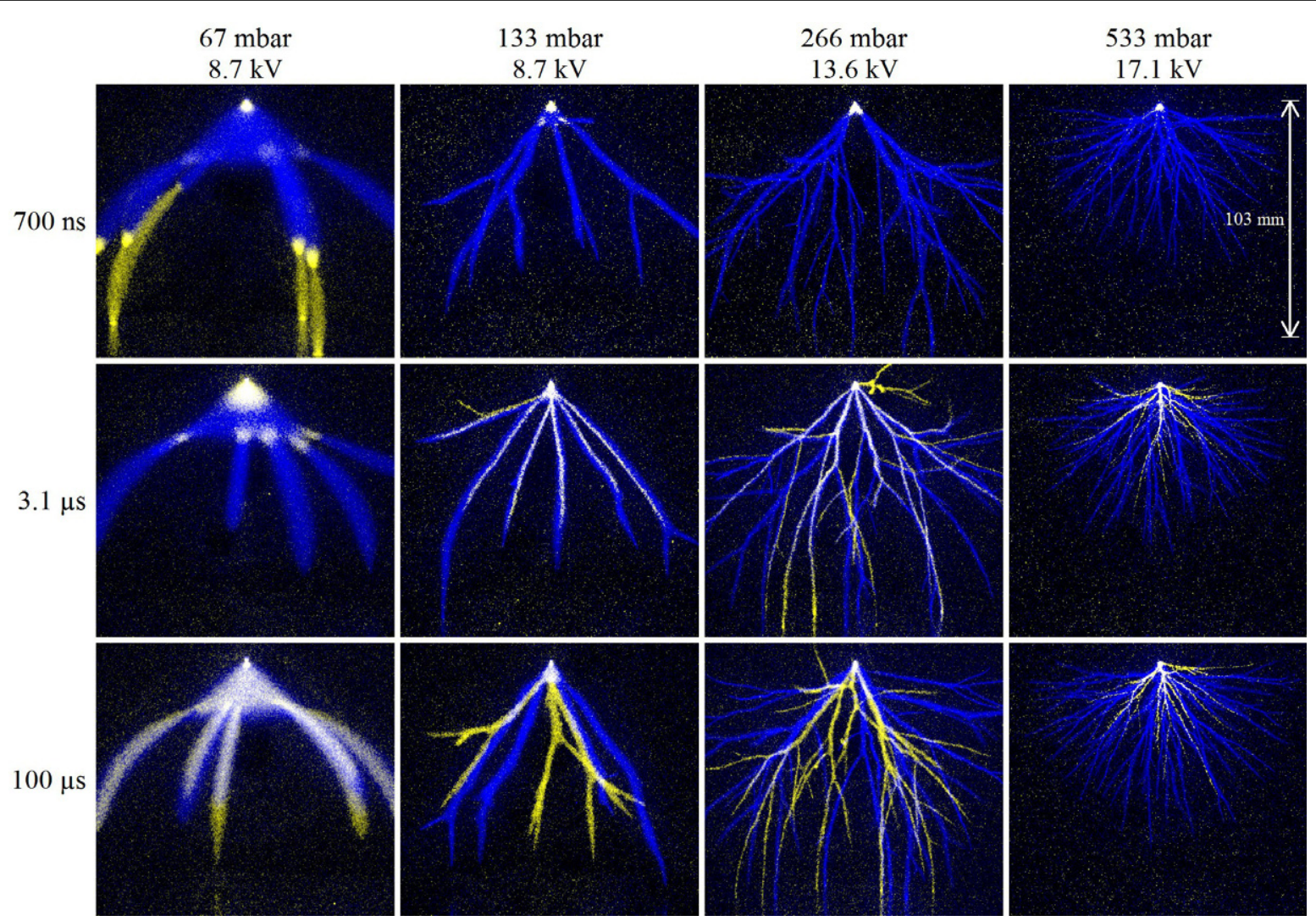

Figure 6. Superimposed discharge-pair images for varying values of $\Delta t$ (indicated on the left), pressure and voltage pulse amplitude (indicated on top). Images taken in artificial air with pulses of $400 \mathrm{~ns}$ length.

When changing voltage and pressure, the effects on the first and second pulse morphologies are in general predictable (see figure 6): at higher voltages more and thicker streamers occur while at higher pressures fewer and thinner streamers occur. Furthermore, the different stages of development occur at lower values of $\Delta t$ for higher pressures and vice versa. More details on the effects of parameter settings on streamer continuation will be given in section 4 .

\subsection{Pure nitrogen}

The same measurements as treated above on air have also been made on pure nitrogen. As was mentioned before, the expected purity in pure nitrogen is about $5 \mathrm{ppm}$. This purity is less than in previous work [15] but still ensures an oxygen concentration that is four to five orders of magnitude lower than in air.

A selection of measurement results obtained in pure nitrogen is given in figure 7. In general, the behaviour of double-pulse streamers in nitrogen is very similar to that in air although there are also some notable differences:

- As usual, streamers in nitrogen are thinner and branch more than streamers in air [15].

- The inception cloud in nitrogen is much smaller than in air, therefore at pressures above $100 \mathrm{mbar}$ it is not possible to distinguish the spots on the edge of the inception cloud.

- The maximum value of $\Delta t$ for which streamer continuation still occurs (stage (i)) is about 1.5-2 times higher than in air. More details will be given in section 4 .
- The value of $\Delta t$ at which new channels start to occur (stages (iii) and (iv)) is much higher in pure nitrogen than in air. For the case of $133 \mathrm{mbar}, 8.7 \mathrm{kV}, 400 \mathrm{~ns}$ new channels following the side of the old ones (stage (iv)) become clearly present around $\Delta t=3 \mu \mathrm{s}$ in air while in pure nitrogen this only starts to occur around $\Delta t=30 \mu \mathrm{s}$.

- Even at very high values of $\Delta t$ (10 ms and above) many second-pulse streamers still follow the rough paths of firstpulse streamers (stage (v)). In air this only occurs up to $\Delta t=1 \mathrm{~ms}$ and in general is less obvious. At $\Delta t=40 \mathrm{~ms}$ in nitrogen the second discharge seems independent of the first.

One thing not shown in figure 7, but very obvious in the total set of measurement images, is the large variation from pulse pair to pulse pair in pure nitrogen: the value of $\Delta t$ at which a certain phenomenon occurs can vary by about a factor of 2 between pulse pairs while in air the behaviour is much more stable. This makes a quantitative study of these phenomena in nitrogen more difficult than in air. Nevertheless, it is still possible to see the trends of streamer morphology as a function of time, but quantitative measurements of properties can easily have error margins of $20 \%$ or more.

\subsection{Pure argon}

A third gas we have investigated next to artificial air and pure nitrogen is argon. The expected purity of argon in our measurements is identical to that of nitrogen. Because streamer discharges in argon can easily lead to the occurrence 

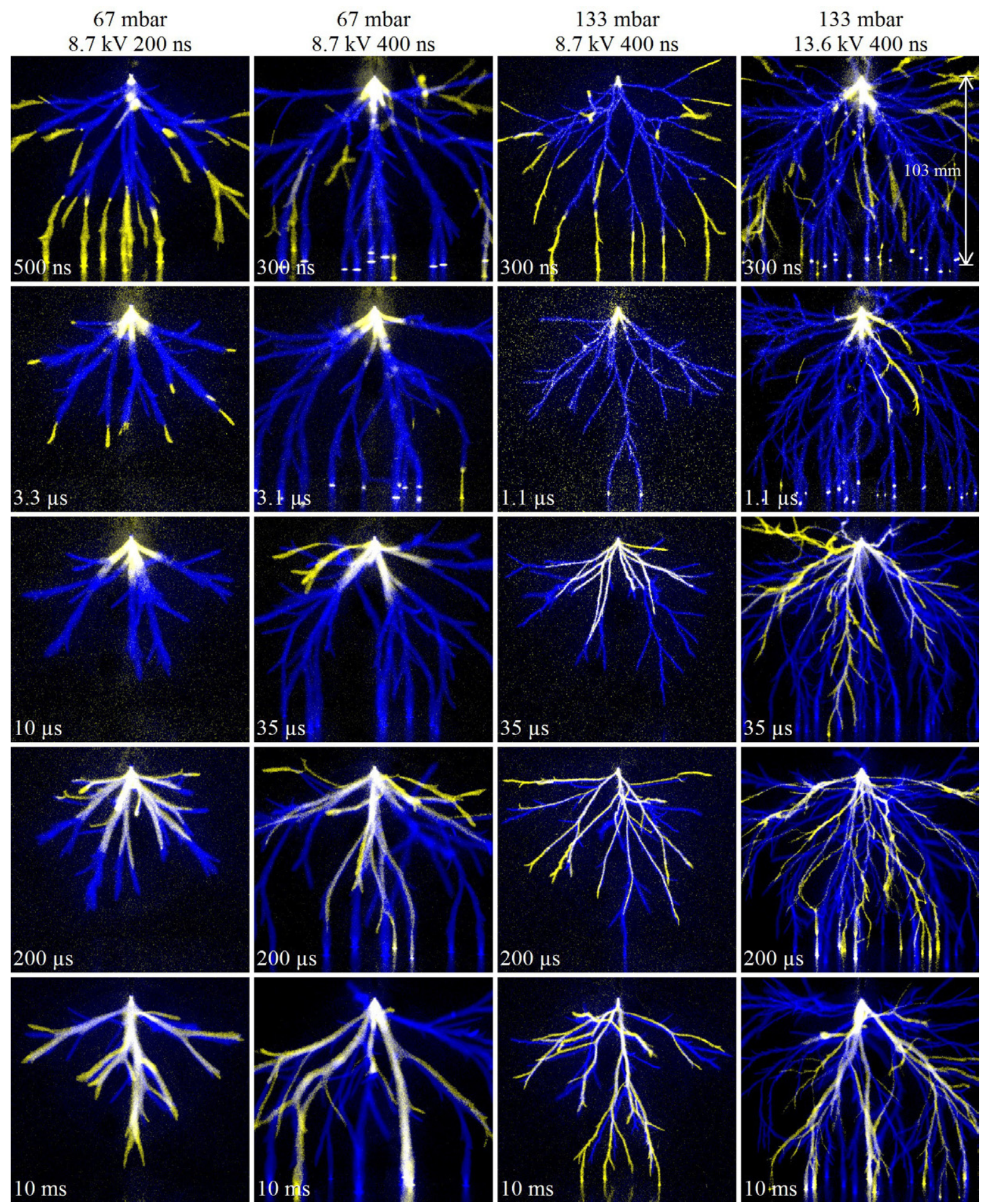

Figure 7. Superimposed discharge-pair images for varying values of $\Delta t$ (indicated on the images), pressure and voltage pulse amplitude and length (indicated on top). Images taken in pure nitrogen.

of a spark, the applied voltages and pulse durations were limited and the lowest pressure used was 133 mbar. This prevents the occurrence of a spark that can potentially damage the electrodes as well as the intensifier in the ICCD cameras.

An overview of double-pulse discharges in $133 \mathrm{mbar}$ argon with a pulse of $8.7 \mathrm{kV}$ amplitude and $200 \mathrm{~ns}$ length is given in figure 8 . The double-pulse discharges in argon have many similarities with discharges in air and nitrogen; however there are again some notable differences:
- The continuation of first-pulse streamers during the second pulse (stage (i)) takes much longer. While in air and nitrogen the maximum value of $\Delta t$ for continuation under similar conditions is less than $2 \mu \mathrm{s}$, in argon it is about $15 \mu \mathrm{s}$. A more detailed comparison will be given in section 4 .

- The decay of excited argon-levels is slower than the decay of the dominant emitting channels in nitrogen (partly due to energy-pooling by metastables). Therefore, the 

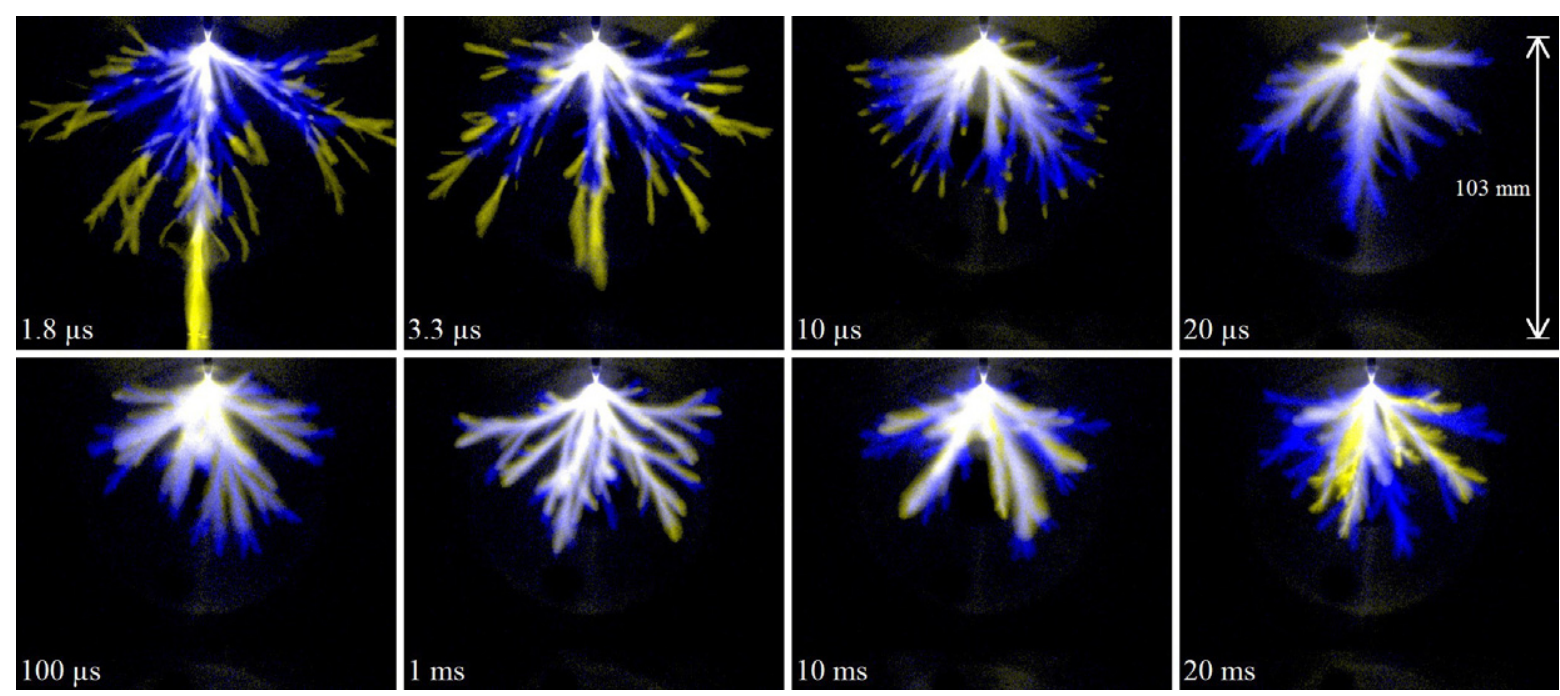

Figure 8. Superimposed discharge-pair images for varying values of $\Delta t$ (as indicated in the images). Images taken in 133 mbar pure argon with pulses of $8.7 \mathrm{kV}$ amplitude and $200 \mathrm{~ns}$ length.

old channels keep emitting light, even when no second pulse is given. This means that for lower values of $\Delta t$ it is impossible to distinguish between light from the still emitting excited species created during the first pulse and newly excited species created during the second pulse. This (partly) explains the large white sections in the centre of the discharge for $\Delta t \lesssim 100 \mu \mathrm{s}$.

- At values of $\Delta t$ between $100 \mu \mathrm{s}$ and $1 \mathrm{~ms}$ the second-pulse streamer channels seem to follow the old channels such as in nitrogen and air (stages (iv) and (v)). However, the large number of channels makes it difficult to tell whether they exactly follow the old paths or, such as in nitrogen and air, hug the edges of the old paths.

- The first-pulse discharges show a very feather-like morphology, as is always visible in argon under similar conditions [5,15]. However, up to $\Delta t=10 \mathrm{~ms}$ the second-pulse streamers (except for the continuing firstpulse streamers) are very smooth. This fits well with previous work on the effects of pre-ionization on pure argon and nitrogen streamer morphology $[1,5]$.

- Between $\Delta t=100 \mu \mathrm{s}$ and $\Delta t=1 \mathrm{~ms}$ the second-pulse channels get more narrow, however, between $\Delta t=1 \mathrm{~ms}$ and $\Delta t=10 \mathrm{~ms}$ they become wider again. The first effect can probably be attributed to a decrease in backgroundionization levels inside the channel, while the second effect may be explained by diffusion of the remaining (low levels) of background ionization.

\section{Duration of streamer continuation}

In a more quantitative study of the important timescales in our experiment, we have measured the maximum value of $\Delta t$, for which the continuation of streamers is still observed, or, in other words, the value of $\Delta t$ determining the border between stages (i) and (ii). We label this quantity $\Delta t_{\mathrm{cm}}$. It was measured as a function of pressure for all gases involved and as a function of oxygen concentration in nitrogen-oxygen mixtures at a fixed pressure. Because the continuation does not

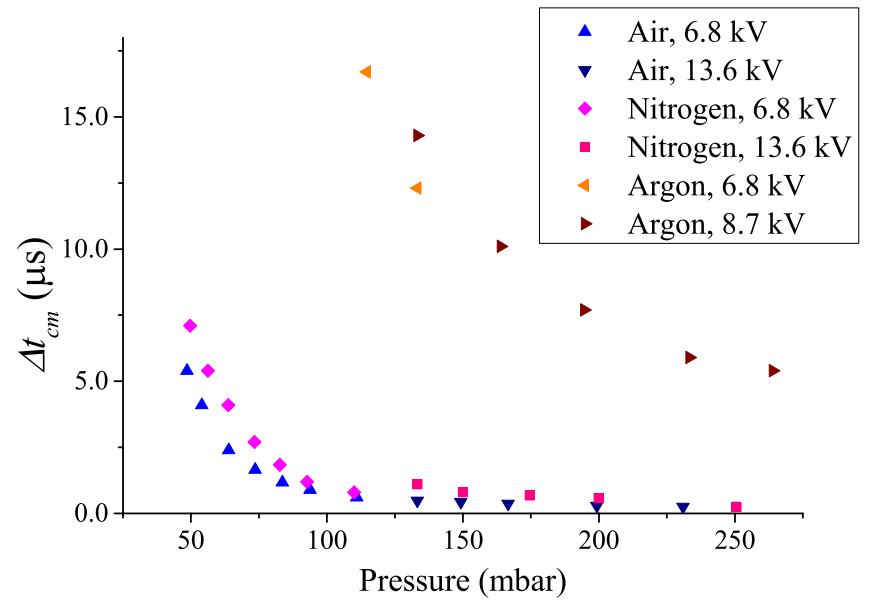

Figure 9. Maximum pulse-to-pulse interval for which continuation still occurs $\left(\Delta t_{\mathrm{cm}}\right)$ in artificial air, pure nitrogen and pure argon as a function of gas pressure for different pulse voltages as indicated. All measurements were made with a pulse length of $300 \mathrm{~ns}$.

stop instantly at a certain $\Delta t, \Delta t_{\mathrm{cm}}$ is defined as the value of $\Delta t$ for which in $50 \%$ of the images some form of continuation is visible.

Similar parameters for the occurrence of other phenomena as a function of $\Delta t$ have also been considered, most notably the time when the first new streamers start to occur. However, the jitter of these phenomena (that happen for larger $\Delta t$ ) can be as large as a factor of 10, especially in pure nitrogen. This makes it nearly impossible to make a coherent data set of such phenomena.

Measured values of $\Delta t_{\mathrm{cm}}$ as a function of pressure for three different gases are plotted in figure 9. It was not possible to measure $\Delta t_{\mathrm{cm}}$ over the entire pressure range with the same voltage pulse amplitude, at lower pressures a very high voltage pulse would lead to streamers that cross the gap during the first pulse and therefore no continuation would be visible. A very low voltage pulse at higher pressures would lead to no or very short and thin streamers where continuation is difficult to 
see. Therefore, the applied voltage pulse was varied over the pressure range, as indicated in the figure.

As could already be concluded from the images shown in the previous section, $\Delta t_{\mathrm{cm}}$ is much larger for argon than for nitrogen or artificial air.

In order to avoid the effects of changing the reduced electric field $(E / n)$ too much we have also measured $\Delta t_{\mathrm{cm}}$ in artificial air and pure nitrogen as a function of pressure while varying the applied voltage $V_{\max }$ so that $V_{\max } / p$ remains constant. We assume constant temperature so that $n$ scales with $p$ according to the ideal gas law. Note that this does not imply that everything scales perfectly with $V_{\max } / p$ but just that the total streamer length ${ }^{5}$ remains roughly constant and that streamer inception is ensured. In this way it is possible to measure $\Delta t_{\mathrm{cm}}$ over a large pressure range without the need for abrupt changes in applied voltage.

Unsurprisingly, in the measurements presented in figure 9 $\Delta t_{\mathrm{cm}}$ increases with decreasing pressure for all three gases. The most obvious cause of the stopping of streamer continuation is a decrease in streamer channel conductivity by various electron loss processes. Many of the rate-limiting reactions involved in the electron loss processes are three particle reactions (involving an electron or an ion and two neutral particles) that scale with $1 / n^{2}$, so we can expect that $\Delta t_{\mathrm{cm}}$ also scales with $1 / n^{2}$ with $n$ the neutral density. Therefore, we have plotted $\Delta t_{\mathrm{cm}}$ in artificial air and nitrogen as a function of $1 / p^{2}$ in figure 10 . In section 5.2 we will treat the relevant electron loss reactions in more detail.

As was expected we can observe a linear relation between $\Delta t_{\mathrm{cm}}$ and $1 / p^{2}$, although the fitted lines do not exactly cross zero. Unfortunately, because of the tendency of argon discharges to form a spark, only a limited pressure range could be explored and it was not possible to measure $\Delta t_{\mathrm{cm}}$ for constant $V_{\max } / p$.

\subsection{Oxygen concentration effects}

Figures 9 and 10 show that although $\Delta t_{\mathrm{cm}}$ is larger for nitrogen than for artificial air, the difference is relatively small, especially in comparison with argon. However, if the value in $\Delta t_{\mathrm{cm}}$ would only be influenced by electron attachment to molecular oxygen, we would expect an increase in $\Delta t_{\mathrm{cm}}$ of at least a few orders of magnitude between air and pure nitrogen. To investigate this in more detail, we have measured and plotted $\Delta t_{\mathrm{cm}}$ as a function of oxygen concentration. This was done by starting with artificial air and then repeatedly replacing $50-70 \%$ of the gas in the vessel with pure nitrogen, always returning to the starting pressure to measure $\Delta t_{\mathrm{cm}}$. The results of this procedure are plotted in figure 11.

We observe that when we decrease the oxygen concentration of artificial air at 133 mbar by mixing in more and more pure nitrogen, $\Delta t_{\mathrm{cm}}$ increases as expected. However, this effect reaches a maximum at an oxygen concentration of about $0.2 \%$. For lower oxygen concentrations $\Delta t_{\mathrm{cm}}$ decreases again and stabilizes around $0.01 \%$ oxygen. A similar

\footnotetext{
5 Streamer length depends here on streamer velocity and pulse duration. Because streamer velocity scales with $E / n$, the streamer length remains roughly constant for a given pulse duration when $V_{\max } / p$ is kept constant.
}

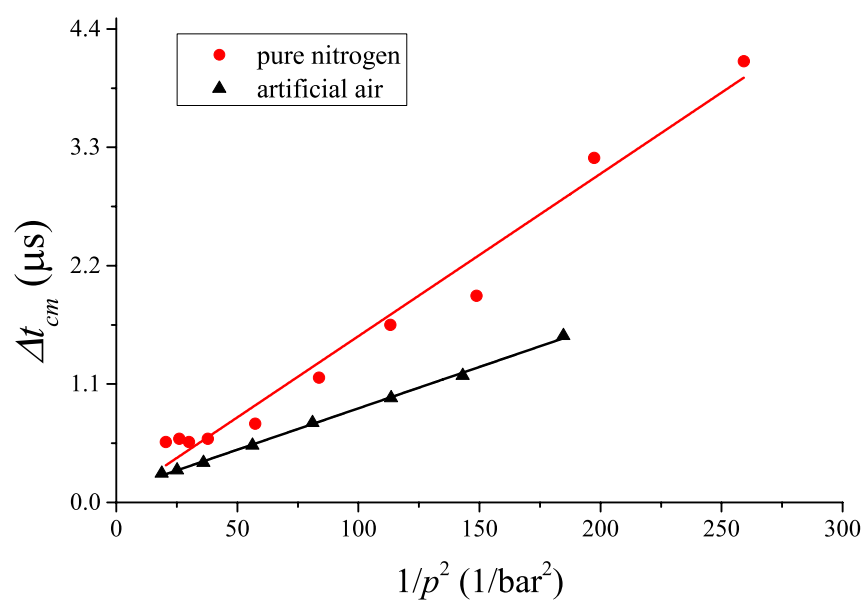

Figure 10. $\Delta t_{\mathrm{cm}}$ in artificial air and nitrogen as a function of $1 / p^{2}$ with $V_{\max } / p$ kept constant at 77.7 and $74.5 \mathrm{~V} \mathrm{mbar}^{-1}$ for nitrogen and air, respectively. The voltage pulse length was kept at $200 \mathrm{~ns}$ for both gases. For both gases a linear fit is included.

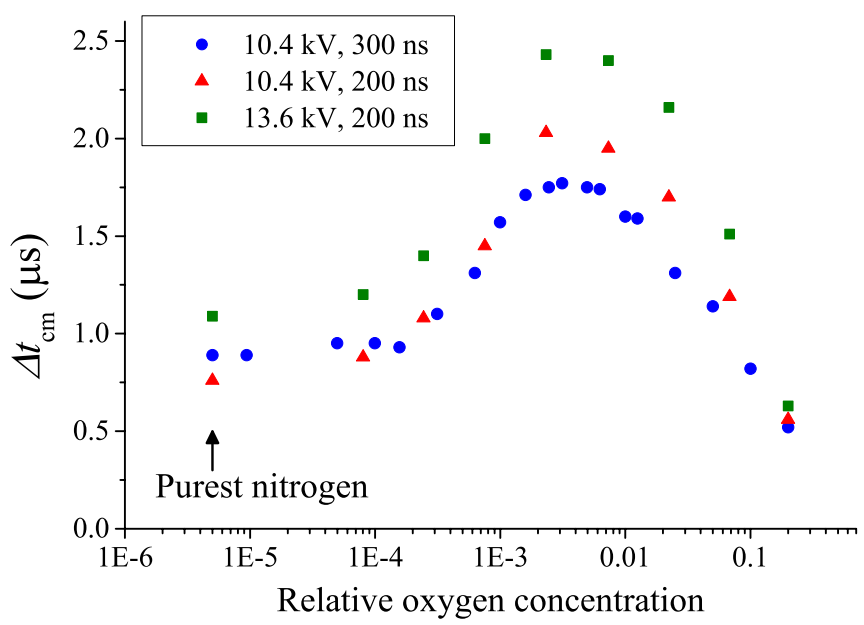

Figure 11. $\Delta t_{\mathrm{cm}}$ as a function of oxygen concentration for different oxygen-nitrogen mixtures. All measurements were made at 133 mbar with the pulse amplitudes and durations as indicated. The points drawn at $5 \mathrm{ppm}$ oxygen concentration are actually measured in pure nitrogen gas (within experimental limitations).

effect has also been found in other streamer measurements: Ono and Oda [18] observed a maximum in time-integrated discharge current for oxygen concentrations (in nitrogen) around $0.2-1.0 \%$, not far from the maximum found here although they measured at 1000 mbar instead of our 133 mbar.

Note that although we have calculated from the leak-rate that the amount of impurities in our purest nitrogen is less than $5 \mathrm{ppm}$, we cannot be entirely sure. It is possible that leaks or impurities in our gas-handling system lead to higher impurity levels. This would mean that the leftmost points in figure 11 shift to the right and the plateau below $0.01 \%$ oxygen could be an artefact of our set-up.

\section{Modelling}

The development of the discharge during the second pulse depends on density and distribution of various species 
(electrons, ions, metastables, etc) left over from the firstpulse discharge. If we fix a computational cell inside of the discharge, neglect drift and diffusion of the species and follow only the temporal dynamics, we can apply zero-dimensional modelling to study the left overs of the first-pulse discharge. By varying the $\mathrm{N}_{2}: \mathrm{O}_{2}$ ratio we can then simulate experimental results from sections 3.1 and 3.2.

\subsection{Model description}

A zero-dimensional model is used to describe the dynamics of species

$$
\frac{\mathrm{d}\left[n_{i}\right]}{\mathrm{d} t}=S_{i},
$$

where the source term $S_{i}$ is the total rate of production or destruction of the species $i$ in various processes; it depends on the local electric field. A modification of the kinetic file for $\mathrm{N}_{2}-\mathrm{O}_{2}$ mixtures from ZDPlasKin $[19,20]$ is used that consists of 650 reactions (listed in the online supplementary data (stacks.iop.org/PSST/23/025008/mmedia)) of the 53 species and states listed in table 1 . The plasma-chemical processes in $\mathrm{N}_{2}-\mathrm{O}_{2}$ mixtures are mainly taken from [21]. Constant rates for electron-neutral interactions are calculated using the BOLSIG+ solver that is contained in the package [22]. The list of species and reactions was automatically converted into the system of ordinary differential equations (1) and solved numerically using the ZDPlasKin tool. The QtPlaskin visualization software was used for the analysis of the results [23].

In order to run ZDPlasKin, the calculation of the electric field has to be brought into the structure of equation (1), but the field profile should also be consistent with the local electron density. We have solved this problem by 1 . neglecting the leading edge of the ionization front, and by 2 . using the conservation of the total current:

$$
\nabla \cdot\left(\varepsilon_{0} \partial_{t} E+j\right)=0
$$

where $j$ is the electric current; in our case it is $j=-e j_{\mathrm{e}}$ with

$$
j_{e}=-\mu(|E|) E n_{\mathrm{e}},
$$

where e, $\mu(|E|)$ and $n_{\mathrm{e}}$ are the charge, electron mobility in $\mathrm{N}_{2}$ and density of electrons, respectively. Electron mobilities are obtained from $[24,25]$, where the multiterm solution of Boltzmann's equation was used. If we approximate the weakly curved front by a planar front [26] and if we assume that the electric field in the non-ionized region does not change in time $\left(\partial_{t} E=0\right.$ where $\left.j=0\right)$, i.e. in zero-dimensional configuration, we obtain

$$
\frac{\mathrm{d} E}{\mathrm{~d} t}=\frac{\varepsilon_{0}}{e} \mu(|E|) E n_{\mathrm{e}} .
$$

As said above, we neglect the leading edge of the front and start integrating at the maximum of the electric field. As [27] reviews, the maximal electric fields in simulated positive streamers in STP air vary between 120 and $180 \mathrm{kV} \mathrm{cm}^{-1}$, so we use $150 \mathrm{kV} \mathrm{cm}^{-1}$. This scales to $E(0)=20 \mathrm{kV} \mathrm{cm}^{-1}$ at 133 mbar according to the similarity laws that approximate the
Table 1. Species considered in the model.

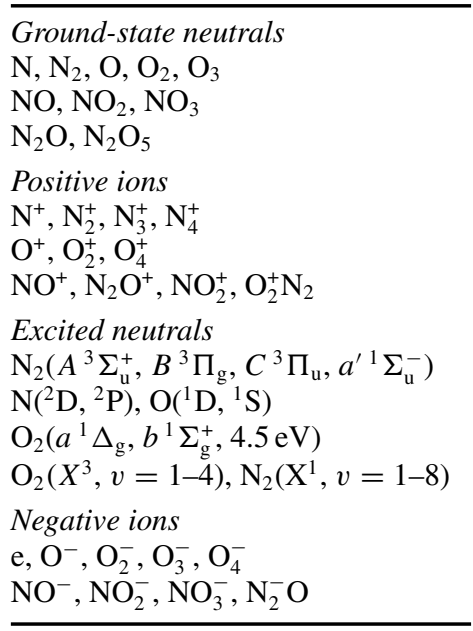

fast processes in streamer heads very well. In the simulations by Naidis [27] we find an electron density of $5.3 \times 10^{12} \mathrm{~cm}^{-3}$ where the electric field is maximal and scale this to $133 \mathrm{mbar}$ to use as our initial electron density $n_{\mathrm{e}}(0)=7.1 \times 10^{11} \mathrm{~cm}^{-3}$. We initialize the densities of the positive ions $\mathrm{N}_{2}^{+}$and $\mathrm{O}_{2}^{+}$such that the plasma is neutral $n_{\mathrm{e}}(0)=n_{\mathrm{N}_{2}^{+}}(0)+n_{\mathrm{O}_{2}^{+}}(0)$ while keeping the initial ratio $n_{\mathrm{N}_{2}^{+}}(0): n_{\mathrm{O}_{2}^{+}}(0)=n_{\mathrm{N}_{2}}(0): n_{\mathrm{O}_{2}}(0)$. The initial densities of all other ions, excited species and ground-state neutrals (except $\mathrm{N}_{2}$ and $\mathrm{O}_{2}$ ) are assumed to be zero.

This approximation allows us to integrate equations (1) and (4) together in the form required by ZDPlasKin, and to obtain a decay of the electric field that is consistent with the local conductivity. We integrate the equations until the electric field reaches $0.67 \mathrm{kV} \mathrm{cm}^{-1}$ which is the so-called stability field for positive streamers in air at 133 mbar. We remark that though we have recently criticized this concept in [28], our simulations presented there still show that the average electric field inside a streamer tree reaches such a value. When the stability field is reached, we continue to integrate equation (1), but now in a constant electric field of $0.67 \mathrm{kV} \mathrm{cm}^{-1}$ until the end of the voltage pulse, and then in a vanishing field.

\subsection{Modelling results on streamer continuation}

The modelled development of $n_{\mathrm{e}}$ as a function of time for artificial air, $0.2 \%$ oxygen in nitrogen and pure nitrogen at 133 mbar is given in figure 12. In this figure also the quantity $\Delta t_{\text {probe }}$ is indicated (for the $0.2 \%$ oxygen mixture). This quantity represents the time between the end of the pulse (the moment the field goes to zero) and the moment a certain probe electron density has been reached. We assume that the conductivity of the channel depends only on electron density and that a certain critical conductivity is required for the streamers to continue their old path during the second pulse. Therefore, we can find this critical density by comparing different $\Delta t_{\text {probe }}$ results with the measured $\Delta t_{\mathrm{cm}}$ values. This is done in figure 13. The shape of the $\Delta t_{\mathrm{cm}}$ and $\Delta t_{\text {probe }}$ curves are remarkably similar: both rise quickly from artificial air towards decreasing oxygen content, have a maximum around $0.2 \%$ oxygen, decrease for even lower oxygen concentrations and then level off again around $100 \mathrm{ppm}$ oxygen. This indicates 


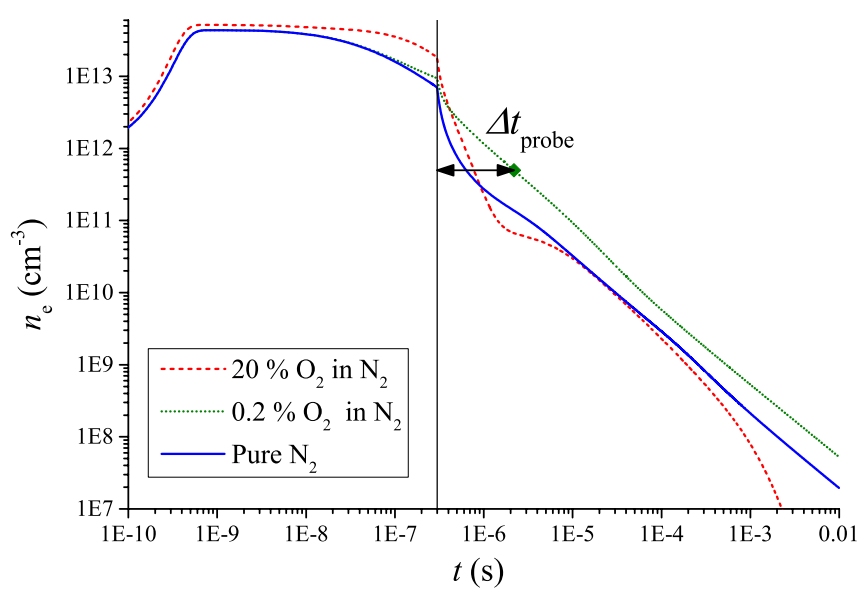

Figure 12. Modelled development of electron density as a function of time for three different gas mixtures at 133 mbar. Pulse duration: $300 \mathrm{~ns}$, initial electron density: $7.1 \times 10^{11} \mathrm{~cm}^{-3}$, maximum electric field on streamer tip: $20 \mathrm{kV} \mathrm{cm}^{-1}$, field in streamer channel:

$0.67 \mathrm{kV} \mathrm{cm}^{-1}$. The end of the pulse is indicated with the vertical line and the quantity $\Delta t_{\text {probe }}$ is indicated for the $0.2 \%$ oxygen mixture with a probing density of $5 \times 10^{11} \mathrm{~cm}^{-3}$.

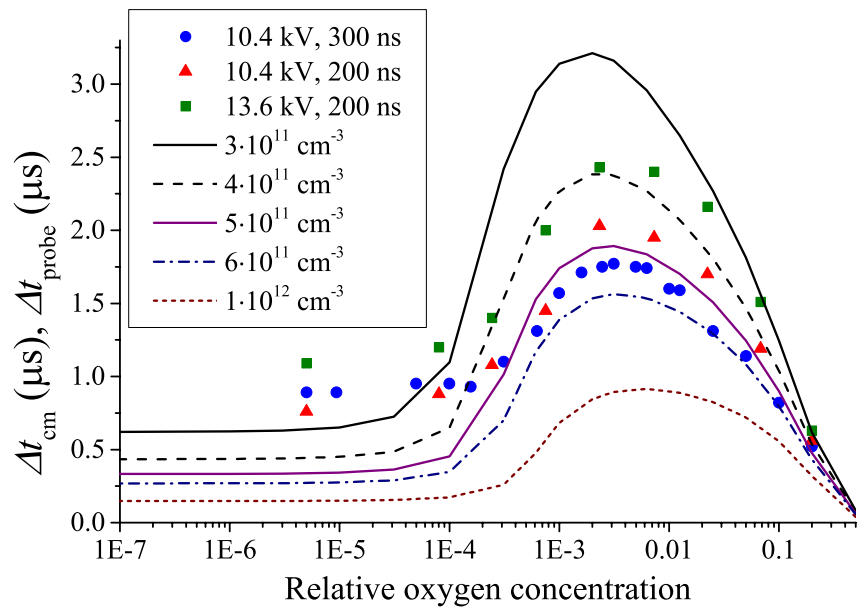

Figure 13. Measured data from figure 11 combined with modelling results of $\Delta t_{\text {probe }}$ with varying probe electron densities as indicated in the legend for the conditions from figure 12.

that the measured curves are no artefact of the measurement method, but can be explained by plasma-chemical processes. The modelling results shown for $0.1 \mathrm{ppm}$ oxygen concentration are virtually identical to results in pure nitrogen.

Note that except for the chosen electron density probing values, no fitting parameters were used in the model. Therefore, even this relatively simple, zero-dimensional model with rate coefficients that can still have large uncertainties is able to predict the shape of $\Delta t_{\mathrm{cm}}$. Only at oxygen concentrations below $100 \mathrm{ppm}$ we observe that the model results are always below the measured results. From the comparison of the model and measurement results we can conclude that the electron density required for continuation of old streamer paths is around $5 \times 10^{11} \mathrm{~cm}^{-3}$ under the conditions presented here.

The shape of the $\Delta t_{\text {probe }}$ and $\Delta t_{\mathrm{cm}}$ curves can be understood by studying the various electron loss mechanisms. In figure 14 the cumulative electron losses by the eight

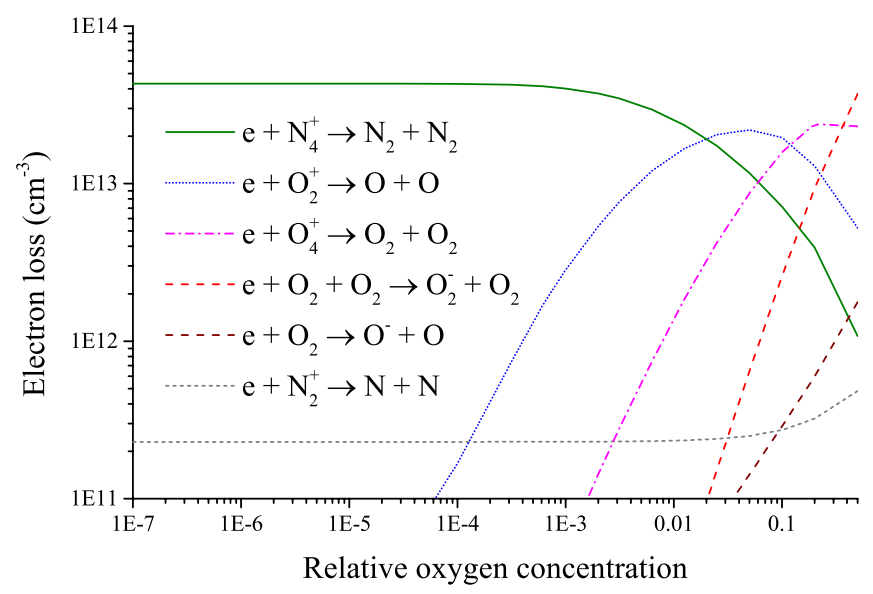

Figure 14. Modelled cumulative electron losses by the eight dominant electron loss processes as a function of oxygen concentration for the conditions from figure 12. Losses are integrated over time between the start of the simulation and the moment $n_{\mathrm{e}}$ reaches a probing value of $5 \times 10^{11} \mathrm{~cm}^{-3}$.

dominant electron loss reactions are plotted as a function of oxygen concentration up to the moment that $n_{\mathrm{e}}=5 \times$ $10^{11} \mathrm{~cm}^{-3}$. In this figure the dissociative attachment reactions with $\mathrm{N}_{2}^{+}$and $\mathrm{O}_{2}^{+}$leading to ground-state atoms are grouped with similar reactions leading to $\mathrm{O}\left({ }^{1} \mathrm{D}\right)$ and $\mathrm{N}\left({ }^{2} \mathrm{D}\right)$ states although they are calculated separately in the actual model.

The figure shows that at low oxygen concentrations (below about $2 \%$ ) the electron loss is dominated by dissociative recombination with $\mathrm{N}_{4}^{+}$. At high oxygen concentrations the electron loss is dominated by electron attachment to molecular oxygen and by dissociative recombination with $\mathrm{O}_{4}^{+}$. All these three processes are relatively fast and thereby can explain the fast decrease in conductivity at high and low oxygen concentrations. However, at intermediate oxygen concentrations, a significant part of the electrons (the major part between $2 \%$ and $12 \%$ oxygen) are lost through dissociative recombination with $\mathrm{O}_{2}^{+}$, which is a much slower process than the dominant electron loss reactions at high and low oxygen concentrations.

In air, the main pathway of positive ions is as follows:

$$
\mathrm{N}_{2}^{+} \Rightarrow \mathrm{N}_{4}^{+} \Rightarrow \mathrm{O}_{2}^{+} \Rightarrow \mathrm{O}_{4}^{+},
$$

as described by Aleksandrov and Bazelyan [29] and also found in our model results. In this case the electron loss is dominated by the fast dissociative recombination with $\mathrm{O}_{4}^{+}$as was discussed above. At high nitrogen and oxygen concentrations this total pathway including recombination is very fast. At low oxygen concentrations the pathway stops at $\mathrm{N}_{4}^{+}$, which also recombines rapidly with free electrons. However, at intermediate oxygen concentrations there is enough oxygen to form $\mathrm{O}_{2}^{+}$but not enough to form reasonable quantities of $\mathrm{O}_{4}^{+}$. As, at $133 \mathrm{mbar}$, the recombination rate of electrons with $\mathrm{O}_{2}^{+}$is much lower than with $\mathrm{O}_{4}^{+}$, the total recombination rate is lower at these intermediate concentrations, which is exactly what we observe in our $\Delta t_{\mathrm{cm}}$ measurements. This is supported by the modelled development of the major ionic species as is plotted in figure 15 .

As was shown in section 4 , when $V_{\max } / p$ is kept constant we observe an almost linear relation between $\Delta t_{\mathrm{cm}}$ and $1 / p^{2}$. 


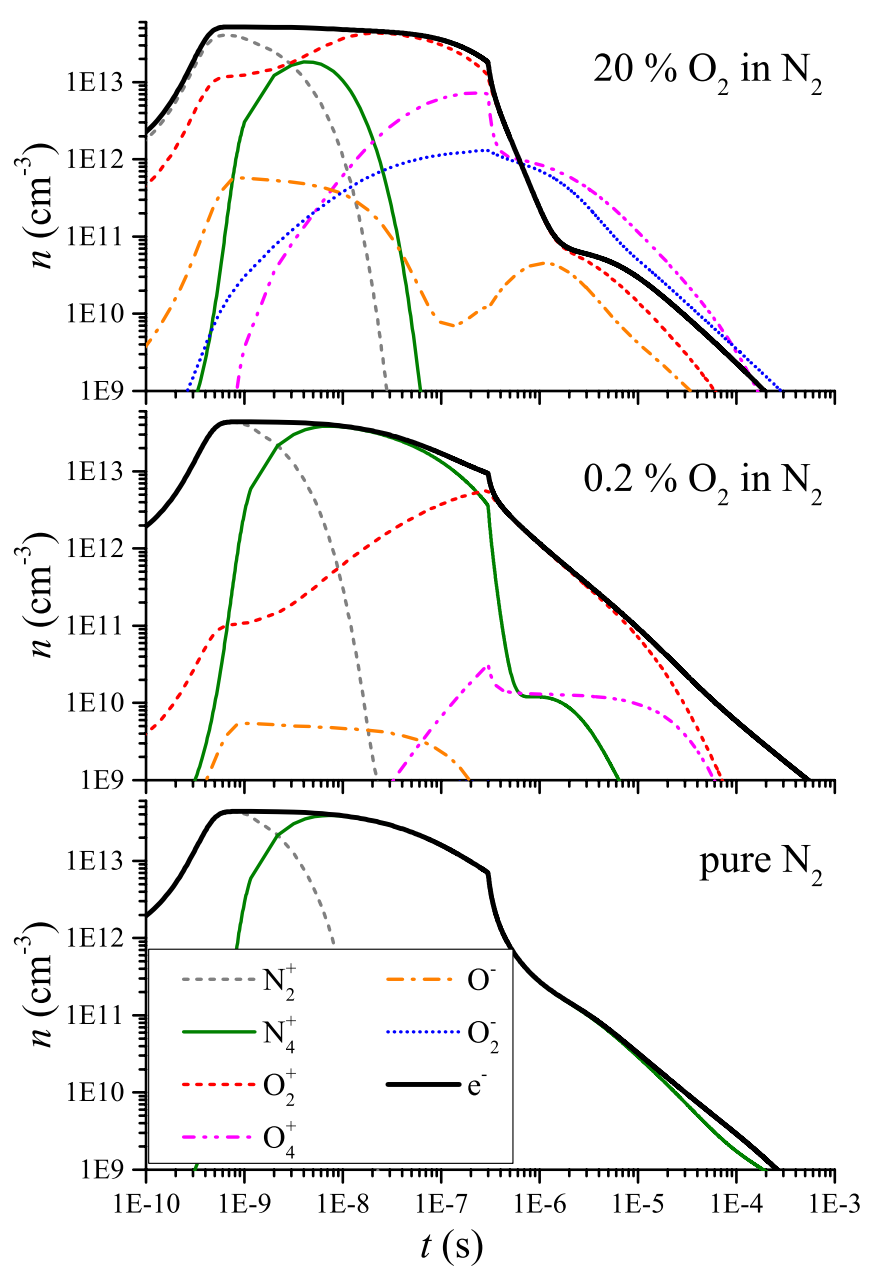

Figure 15. Modelled development of the densities of the major ionic species and the electrons in three different gas mixtures for the conditions indicated in figure 12 .

This was attributed to three-body processes responsible for the electron loss. This is not immediately obvious from the list of dominant reactions in figure 14 where the attachment of electrons to molecular oxygen is the only three-body reaction. However, also the creation of $\mathrm{N}_{4}^{+}$and $\mathrm{O}_{4}^{+}$requires three-body reactions. The dominant production reactions for these species are

$$
\begin{aligned}
& \mathrm{N}_{2}^{+}+\mathrm{N}_{2}+\mathrm{N}_{2} \rightarrow \mathrm{N}_{4}^{+}+\mathrm{N}_{2}, \\
& \mathrm{O}_{2}^{+}+\mathrm{O}_{2}+\mathrm{M}_{2} \rightarrow \mathrm{O}_{4}^{+}+\mathrm{M}_{2},
\end{aligned}
$$

with $\mathrm{M}$ either $\mathrm{O}$ or $\mathrm{N}$. Therefore it is not surprising that $\Delta t_{\mathrm{cm}}$ scales approximately with $1 / p^{2}$.

However, if we look at the calculated values of $\Delta t_{\text {probe }}$ plotted in figure 16, there is no perfect linear relation between $\Delta t_{\text {probe }}$ and $1 / p^{2}$. This may be caused by the constant electric fields that were applied in the model and that lead to lower than proportional $\Delta t_{\text {probe }}$ for increasing $1 / p^{2}$. This can also be observed from the accompanying measurement results at fixed applied voltage (triangles in the figure). Unfortunately, the model did not converge for pressures below 100 mbar.

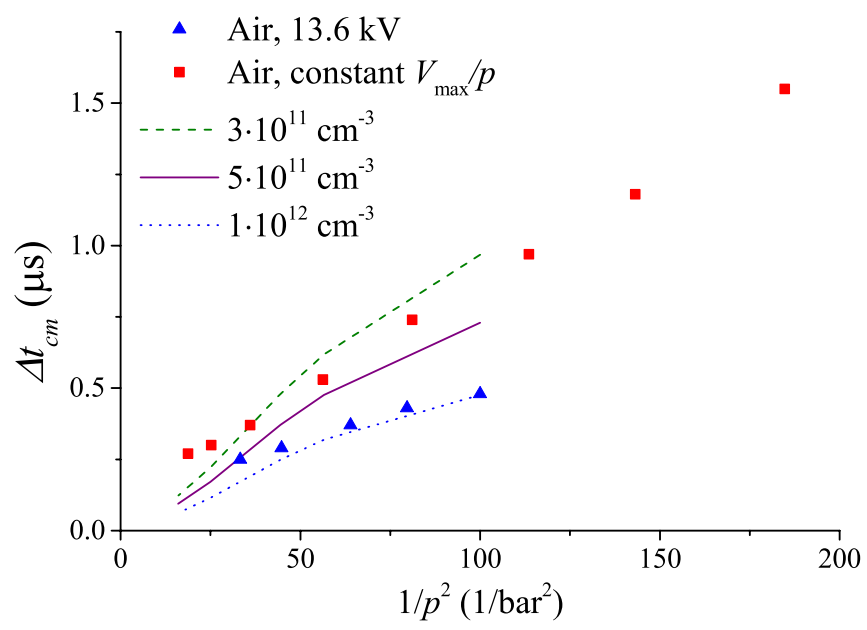

Figure 16. Measured $\Delta t_{\mathrm{cm}}$ as a function of $1 / p^{2}$ in artificial air with $13.6 \mathrm{kV}, 300 \mathrm{~ns}$ pulses (triangles), $\Delta t_{\mathrm{cm}}$ at constant $V_{\max } / p$ (squares, same data as plotted in figure 10) compared with modelling results with $250 \mathrm{~ns}$ pulse lengths for different probe electron densities.

\subsection{Other modelling results}

The experiments have shown that in 133 mbar artificial air or pure nitrogen, new streamers start to occur around $\Delta t \approx$ $2.5 \mu \mathrm{s}$ and $\Delta t \approx 35 \mu \mathrm{s}$, respectively. From the modelled development of $n_{\mathrm{e}}$ (see figure 12) we cannot fully understand these timescales: there is no probing value of $n_{\mathrm{e}}$ that gives such a difference in $\Delta t_{\text {probe }}$. In general, the values of $\Delta t_{\text {probe }}$ for artificial air and pure nitrogen are quite similar for any $n_{\mathrm{e}}$ for probing times below a few hundred microseconds. When looking at the total ionization density as a function of time (i.e. the sum of electrons and negative ions) the results deviate more from the observations: the ionization degree is higher in air than in nitrogen at any time after the pulse. This is because in air the ionization density is not given by only the electron density but the density of the negative ions has to be included which increases the ionization density significantly. Therefore, we can conclude that the occurrence of new streamers cannot be directly linked to either electron or ionization density.

In section 3.2 we observed that in pure nitrogen streamers can follow the path of their predecessors even at $\Delta t \geqslant 10 \mathrm{~ms}$ while in artificial air this is only observed up to $\Delta t \approx 1 \mathrm{~ms}$ and even then this occurs much less often. This fits well with the modelled $n_{\mathrm{e}}$ development which in air starts to drop much quicker than in nitrogen after about $t=100 \mu \mathrm{s}$. This also holds for the total ionization degree, which, although (in air) is higher than the electron density, shows the same trend and drops below the one for nitrogen at about $1.3 \mathrm{~ms}$.

All mentioned numbers are for a pressure of 133 mbar and room temperature. Of course electrons will also be lost through (ambipolar) diffusion, which will start to play a role around millisecond timescales and is not incorporated in our model. 


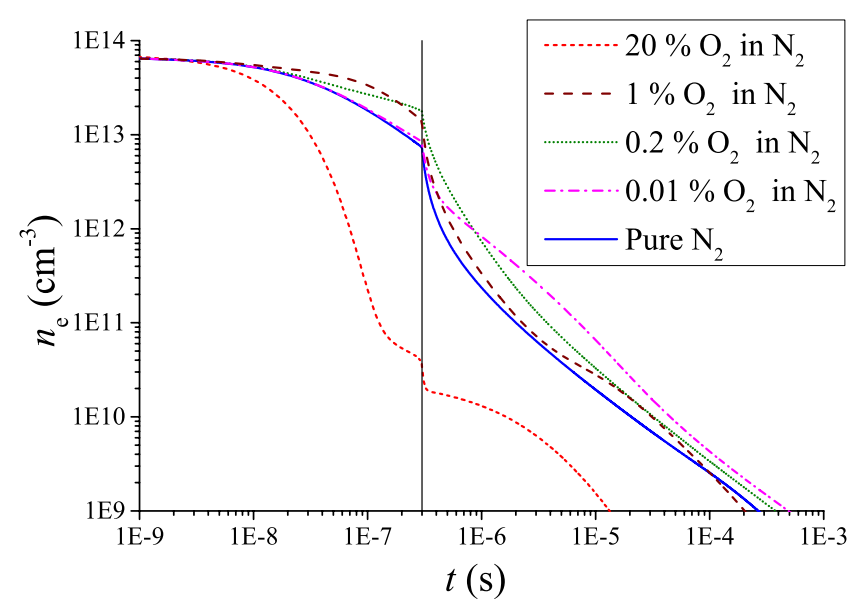

Figure 17. Modelled development of electron density as a function of time for five different gas mixtures at 1000 mbar. Pulse duration: $300 \mathrm{~ns}$, initial electron density: $5.3 \times 10^{12} \mathrm{~cm}^{-3}$, maximum electric field on streamer tip: $150 \mathrm{kV} \mathrm{cm}^{-1}$, field in streamer channel: $5 \mathrm{kV} \mathrm{cm}^{-1}$. The end of the pulse is indicated with the vertical line.

\section{Summary and conclusions}

\subsection{Continuation of old paths}

In this work, we have shown that the application of two consecutive pulses is a valuable tool to improve our understanding of streamer discharges and the interaction between repetitive discharges. We have found that it is possible to 'reignite' a streamer with a second high voltage pulse so that it continues its old path. This is only possible for relatively short durations ( $\left.\Delta t \leqslant \Delta t_{\mathrm{cm}}\right)$ after the first pulse. The measured values of $\Delta t_{\mathrm{cm}}$ are between 0.5 and $15 \mu \mathrm{s}$ at 133 mbar depending on the gas mixture. In nitrogen-oxygen mixtures we have observed that $\Delta t_{\mathrm{cm}}$ exhibits a maximum around $0.2 \%$ oxygen in nitrogen. For lower and higher oxygen concentrations $\Delta t_{\mathrm{cm}}$ decreases. When modelling the decrease in electron density after a streamer discharge we find the same pattern. For probe electron densities around $5 \times 10^{11} \mathrm{~cm}^{-3}$ the decay times fit very well with our measurements. Therefore we conclude that the maximum in $\Delta t_{\mathrm{cm}}$, and the continuation of old paths in general, can be explained by a loss of conductivity in the streamer channel due to the loss of free electrons and that, under our conditions, the minimum $n_{\mathrm{e}}$ for continuation is about $5 \times 10^{11} \mathrm{~cm}^{-3}$ at 133 mbar.

The explanation for the maximum in $\Delta t_{\mathrm{cm}}$, or minimum in total electron loss rate, is that at the oxygen concentrations of this maximum the major loss process is recombination with $\mathrm{O}_{2}^{+}$, while at lower/higher oxygen concentrations the electron loss is dominated by recombination with $\mathrm{N}_{4}^{+}$and $\mathrm{O}_{4}^{+}$, respectively, combined with attachment at high oxygen concentrations. These three processes are all faster than recombination with $\mathrm{O}_{2}^{+}$which explains the maximum in $\Delta t_{\mathrm{cm}}$.

Höft et al [30] have found an increase in discharge decay time when changing the oxygen concentration in nitrogen from $20 \%$ to $0.1 \%$ in a pulsed atmospheric pressure dielectric barrier discharge. This is in line with our observations. Figure 17 shows that the development of $n_{\mathrm{e}}$ at atmospheric pressure follows a similar general trend as observed at lower pressures: $n_{\mathrm{e}}$ decays fastest for artificial air, is slower for lower oxygen concentrations, but is again somewhat faster for pure nitrogen. However, the difference between air and pure nitrogen is much larger than at lower pressures due to the much faster three-body attachment at atmospheric pressure. Also the maximum in $\Delta t_{\text {probe }}$ for probe densities below $1 \times 10^{12} \mathrm{~cm}^{-3}$ now occurs at $0.01 \%$ oxygen, instead of $0.2 \%$ as was found at 133 mbar $^{6}$. Höft et al conclude that the decay time increases with decreasing oxygen concentration, and our observations confirm their findings at atmospheric pressure and oxygen concentrations above $0.01 \%$. According to our modelling, an extension of these measurements to higher purity nitrogen should show a maximum in decay time and a decrease when going to very pure nitrogen.

It is probable that this mechanism can also explain the maximum in integrated current density (and light emission) found by Ono and Oda [18] at oxygen concentrations around $0.2-1.0 \%$ in atmospheric pressure point-plane discharges. They explain the increase in current when adding small amounts of oxygen to pure nitrogen by the increased streamer diameter and photo-ionization. Too much oxygen would lead to electron attachment and therefore reduces the current. This is in line with our modelling results, although it disregards the effects of recombination with $\mathrm{O}_{4}^{+}$. The increase in current with small oxygen additions also matches our modelling results at atmospheric pressure (figure 17) which shows the highest electron density during the pulse $(t<300 \mathrm{~ns})$ for oxygen concentrations of $0.2 \%$ and $1 \%$.

Walsh et al $[10,11]$ also presented a similar continuation phenomenon in atmospheric pressure plasma-jets in helium but did not discuss the maximum continuation time as it was limited by the applied voltage pulse length.

An open question is the exact physical mechanism that determines the critical or probing density. It is clear that the electron density at the end of the first voltage pulse is sufficient to let the streamer return to electrical neutrality between voltage pulses. A possible hypothesis would be that the probing density is determined by the Maxwell relaxation time given by the electron density at the beginning of the second pulse. If that time is comparable to the voltage rise time, the streamer can be screened again and continue to grow. However, the Maxwell relaxation times for our conditions are sub-nanosecond at the electron densities expected at this time (at 133 mbar), while the voltage rise time is about $15 \mathrm{~ns}$. For the same reason one would expect the critical density to depend on pressure but figure 16 implies that it is not.

\subsection{Other phenomena}

In addition to the continuation of streamers for short pulseto-pulse intervals, we have observed a few other interesting phenomena at longer pulse-to-pulse intervals. When the interval is long enough to prevent streamer continuation (i.e. the conductivity has dropped enough), the remaining ionization still prevents new streamers to occur. We attribute

\footnotetext{
6 We have calculated the development of $n_{\mathrm{e}}$ at atmospheric pressure for only five oxygen concentrations so the real maximum can be higher or lower than $0.01 \%$ oxygen.
} 
this to the very high electron density which, though too low for continuation, is still high enough to shield the electrode tip and prevent streamer formation. During this period we only observe some re-glowing or secondary streamers in the area of the first discharge, especially at the edge between inception cloud and streamers as well as at the cathode spots for the cases where the first streamers have crossed the gap.

After some time (pulse-pulse intervals of a few to some tens of microseconds, corresponding to electron densities of $10^{10}-10^{11} \mathrm{~cm}^{-3}$ at $133 \mathrm{mbar}$ ) new streamers start to occur. The first of these still avoid the entire area occupied by the first-pulse discharge. For slightly larger values of $\Delta t$ the new streamers penetrate the area of the first-pulse discharge. These new streamers are much thinner than the first-pulse streamers and follow the edges of the paths of the original channels. This is similar to the streamers observed in [2] that also follow the edge of a pre-ionized region. Apparently the streamers cannot enter the area of high leftover electron density of the old channel because they cannot enhance the field there but preferentially follow the intermediate electron density levels present at the edge of these channels.

For still larger values of $\Delta t$ (tens to hundreds of microseconds corresponding to electron densities of $10^{9}-10^{10} \mathrm{~cm}^{-3}$ at $133 \mathrm{mbar}$ ) the new streamers grow in size and start to overlap more with the first-pulse channels. Finally, at even larger values of $\Delta t$ (electron densities below $10^{8} \mathrm{~cm}^{-3}$ at 133 mbar) they become fully independent of the firstpulse channels. This transition is very gradual and in most cases some second-pulse channels still follow a first-pulse channel while others do not. In nitrogen the following of first-pulse streamers occurs much longer than in artificial air. This is caused by the faster loss of electrons in air by electron attachment which, at longer timescales, dominates over recombination as it scales with $n_{\mathrm{e}}$ instead of with $n_{\mathrm{e}} n_{+}$.

All these later-stage phenomena can be explained by the same two mechanisms: streamers prefer and follow elevated levels of electron density but cannot propagate through areas with very high electron density.

\section{Acknowledgments}

SN was supported by FY2012 Researcher Exchange Program between the Japan Society for the Promotion of Science JSPS and The Netherlands' Organisation for Scientific Research NWO, ET by JSPS KAKENHI Grant Number 24560249 and AM by STW-project 10751, part of NWO.

\section{References}

[1] Nijdam S, Wormeester G, van Veldhuizen E M and Ebert U 2011 Probing background ionization: positive streamers with varying pulse repetition rate and with a radioactive admixture J. Phys. D: Appl. Phys. 44455201

[2] Nijdam S, Miermans K, van Veldhuizen E M and Ebert U 2011 A peculiar streamer morphology created by a complex voltage pulse IEEE Trans. Plasma. Sci. 39 2216-17

[3] Shao T, Sun G S, Yan P, Wang J, Yuan W Q, Sun Y H and Zhang S C 2006 An experimental investigation of repetitive nanosecond-pulse breakdown in air J. Phys. D: Appl. Phys. 39 2192-7
[4] Pai D Z, Lacoste D A and Laux C O 2010 Transitions between corona, glow, and spark regimes of nanosecond repetitively pulsed discharges in air at atmospheric pressure J. Appl. Phys. 107093303

[5] Takahashi E, Kato S, Sasaki A, Kishimoto Y and Furutani H 2011 Controlling branching in streamer discharge by laser background ionization J. Phys. D: Appl. Phys. 44075204

[6] Mathew D, Bastiaens H M J, Boller K J and Peters P J M 2007 Effect of preionization, fluorine concentration, and current density on the discharge uniformity in $\mathrm{F}_{2}$ excimer laser gas mixtures J. Appl. Phys. 102033305

[7] Winands G J J, Yan K, Pemen A J M, Nair S A, Liu Z and van Heesch E J M 2006 An industrial streamer corona plasma system for gas cleaning IEEE Trans. Plasma Sci. 34 2426-33

[8] Laroussi M and Akan T 2007 Arc-free atmospheric pressure cold plasma jets: a review Plasma Proces. Polym. $4777-88$

[9] Starikovskiy A and Aleksandrov N 2013 Plasma-assisted ignition and combustion Prog. Energy Combust. Sci. 39 61-110

[10] Walsh J L, Olszewski P and Bradley J W 2012 The manipulation of atmospheric pressure dielectric barrier plasma jets Plasma Sources Sci. Technol. 21034007

[11] Naidis G V and Walsh J L 2013 The effects of an external electric field on the dynamics of cold plasma jets-experimental and computational studies J. Phys. D. Appl. Phys. 46095203

[12] Nijdam S, Moerman J S, Briels T M P, van Veldhuizen E M and Ebert U 2008 Stereo-photography of streamers in air Appl. Phys. Lett. 92101502

[13] Ebert U, Montijn C, Briels T M P, Hundsdorfer W, Meulenbroek B, Rocco A and van Veldhuizen E M 2006 The multiscale nature of streamers Plasma Sources Sci. Technol. 15 S118

[14] Marode E, Djermoune D, Dessante P, Deniset C, Ségur P, Bastien F, Bourdon A and Laux C 2009 Physics and applications of atmospheric non-thermal air plasma with reference to environment Plasma Phys. Control. Fusion 51124002

[15] Nijdam S, van de Wetering F M J H, Blanc R, van Veldhuizen E M and Ebert U 2010 Probing photo-ionization: experiments on positive streamers in pure gases and mixtures J. Phys. D: Appl. Phys. 43145204

[16] Briels T M P, Kos J, van Veldhuizen E M and Ebert U 2006 Circuit dependence of the diameter of pulsed positive streamers in air J. Phys. D: Appl. Phys. 395201

[17] Riousset J A, Pasko V P and Bourdon A 2010 Air-density-dependent model for analysis of air heating associated with streamers, leaders, and transient luminous events J. Geophys. Res.: Space Phys. (1978-2012) 115 A 12321

[18] Ono R and Oda T 2003 Formation and structure of primary and secondary streamers in positive pulsed corona discharge - effect of oxygen concentration and applied voltage J. Phys. D: Appl. Phys. 36 1952-8

[19] Pancheshnyi S, Eismann B, Hagelaar G J M and Pitchford L C Computer code ZDPlasKin (University of Toulouse, LAPLACE, CNRS-UPS-INP, Toulouse, France, 2008) www.zdplaskin.laplace.univ-tlse.fr

[20] Flitti A and Pancheshnyi S 2009 Gas heating in fast pulsed discharges in $\mathrm{N}_{2}-\mathrm{O}_{2}$ mixtures Eur. Phys. J.. Appl. Phys. 4521001

[21] Ferreira C M, Gordiets B F and Osipov A I 2000 Plasma Kinetics in Atmospheric Gases (Springer Series on Atomic, Optical, and Plasma Physics) (Berlin: Springer) 
[22] Hagelaar G J M and Pitchford L C 2005 Solving the Boltzmann equation to obtain electron transport coefficients and rate coefficients for fluid models Plasma Sources Sci. Technol. 14 722-33

[23] Luque A Computer code QPlaskin www.trappa.es/content/ software

[24] Dujko S, Markosyan A H, White R D and Ebert U 2013 High order fluid model for streamer discharges: I. Derivation of model and transport data J. Phys. D: Appl. Phys. 46475202

[25] Dujko S, Ebert U, White R D and Petrović Z L 2011 Boltzmann equation analysis of electron transport in a $\mathrm{N}_{2}-\mathrm{O}_{2}$ streamer discharge Japan. J. Appl. Phys. 50 08JC01

[26] Ebert U et al 2011 Multiple scales in streamer discharges, with an emphasis on moving boundary approximations NonLinearity $24 \mathrm{C} 1$
[27] Naidis G V 2009 Positive and negative streamers in air: velocity-diameter relation Phys. Rev. E 79057401

[28] Luque A and Ebert U 2013 Growing discharge trees with self-consistent charge transport: the collective dynamics of streamers (arXiv:1307.2378)

[29] Aleksandrov N L and Bazelyan E M 1999 Ionization processes in spark discharge plasmas Plasma Sources Sci. Technol. 8285

[30] Höft H, Kettlitz M, Hoder T, Weltmann K D and Brandenburg R 2013 The influence of $\mathrm{O}_{2}$ content on the spatio-temporal development of pulsed driven dielectric barrier discharges in $\mathrm{O}_{2} / \mathrm{N}_{2}$ gas mixtures J. Phys. D: Appl. Phys. 46095202 